\title{
Os Sete Lados do Triângulo: Desenvolvimento do Questionário Multidimensional de Comportamentos Extrarrelacionais (QMCER)
}

\author{
The Seven Sides of the Triangle: Development of the Multidimensional Extra-Relational \\ Behaviors Questionnaire
}

\author{
Thaysa Viegas $^{1}$ e João Manuel Moreira ${ }^{2}$
}

\begin{abstract}
Resumo
A infidelidade nas relações de casal é um acontecimento frequente e com consequências potencialmente gravosas para os envolvidos. Apesar desta importância, existe uma escassez de instrumentos para a sua avaliação psicológica, que importa minorar. Nesse sentido, foi elaborado o Questionário Multidimensional de Comportamentos Extrarrelacionais (QMCER), composto por 6 secções. Análises em componentes principais $(\mathrm{N} \geq 156)$ sustentam a ideia de que o QMCER permite avaliar a ocorrência de 3 tipos de comportamentos extrarrelacionais (envolvimento sexual, envolvimento afetivo e desejo sexual), 7 dimensões associadas às caraterísticas da infidelidade, suas motivações e consequências da mesma, e 4 fatores ligados à motivação para a fidelidade, que se organizam de forma semelhante à postulada pela teoria da autodeterminação. Os valores do coeficiente alfa de Cronbach são geralmente adequados, com exceção de algumas escalas que requerem desenvolvimento futuro. As correlações entre as escalas e com outros instrumentos fornecem dados positivos quanto à validade do QMCER.
\end{abstract}

Palavras-chave: infidelidade, conjugalidade, psicologia evolutiva, vinculação, género

\begin{abstract}
Infidelity in couple relationships is a common event, and one with potentially serious consequences for those involved. In spite of its importance, there is a scarcity of instruments for its psychological evaluation, which it would be important to compensate for. For that purpose, the Questionário Multidimensional de Comportamentos Extrarrelacionais (QMCER; Multidimensional Extra-Relational Behaviors Questionnaire) has been developed, and is composed of 6 sections. Principal components analyses $(\mathrm{N} \geq 156)$ sustain the idea that the QMCER can evaluate the occurrence of 3 types of extra-relational behaviors (sexual involvement, affective involvement and sexual desire), 7 dimensions associated with infidelity characteristics, motivations, and consequences, and 4 factors related to motivations for fidelity, which are organized in a way compatible with self-determination theory. Cronbach alpha values are generally adequate, with the exception of a few scales that need further development. Correlations among the scales and with other instruments provide good indications regarding the validity of the QMCER.
\end{abstract}

Keywords: infidelity, marriage, evolutionary psychology, attachment, gender

Este trabalho foi financiado pela Fundação para a Ciência e a Tecnologia através da BD/61504/2009.

\footnotetext{
${ }^{1}$ Mestre em Psicologia pelo ISPA - Instituto Universitário de Ciências Psicológicas, Sociais e da Vida, doutoranda em Psicologia pela Faculdade de Psicologia da Universidade de Lisboa. E-mail: thaysa@campus.ul.pt

${ }^{2}$ Doutor em Psicologia pela Faculdade de Psicologia da Universidade de Lisboa, professor auxiliar na Faculdade de Psicologia da Universidade de Lisboa. E-mail: joao.moreira@campus.ul.pt
} 


\section{Introdução}

$\mathrm{Na}$ cultura ocidental, as relações monogâmicas assentes no amor romântico têm usualmente como uma das suas regras básicas a exclusividade na intimidade sexual e/ou emocional (Barta \& Kiene, 2005; Boekhout, Hendrick, \& Hendrick, 2003; Fife, Weeks, \& Gambescia, 2008; Magalhães, 2009), o querer ser o único a ser amado pelo outro, com este a ocupar grande parte do nosso tempo, pensamento e atividade (Rubin, 1970). A maioria das pessoas parece considerar-se leal e fiel, tendo a infidelidade como inaceitável (Treas \& Giesen, 2000). No entanto, dado que a ocorrência de comportamentos extrarrelacionais é comum (Hall \& Fincham, 2009), é bem possível que muitas das pessoas que afirmam desaprovar a infidelidade acabem por contribuir para a considerável frequência destes comportamentos (Foster \& Misra, 2013). É sabido que a ocorrência de comportamentos de infidelidade tem um impacto, em regra forte e negativo, na relação de casal (Brown 2001a, 2001b; Hall \& Fincham, 2009), contribuindo para níveis elevados de stress, diminuindo a qualidade e satisfação com a relação conjugal e constituindo um dos principais desencadeadores de crise e de recurso à terapia de casal (Snyder, Baucom, \& Gordon, 2008). Sendo a infidelidade um comportamento com tão grandes consequências para os indivíduos e as famílias, seria desejável que a investigação e a prática clínica se apoiassem em instrumentos de avaliação psicológica devidamente validados e que focassem diferentes aspetos do construto, permitindo obter mais informação relevante para o diagnóstico e a intervenção.

Porém, a investigação sobre infidelidade, no que é do nosso conhecimento, tem adotado uma perspetiva algo simplista na avaliação deste tipo de comportamentos. Na maioria dos casos, a variável é operacionalizada através de questões simples, muitas vezes únicas (e.g., "Teve sexo extramarital nos últimos 2 meses?”; Liu, 2000; Previti \& Amato, 2004; Treas \& Giesen, 2000). Mesmo nos casos em que a investigação procura identificar as causas da infidelidade, tende a utilizar cenários hipotéticos e dados retrospetivos, não recolhendo informação detalhada acerca dos comportamentos de infidelidade, dos seus contextos e motivações (Allen et al., 2005; Hackathorn, Mattingly, Clark, \& Mattingly, 2011; Hall \& Fincham, 2006; mas ver Romero Palencia, Rivera Aragón, \& Díaz Loving, 2007 como uma excepção). $\mathrm{Na}$ nossa opinião, este subdesenvolvimento dos métodos de avaliação nos domínios do comportamento extramarital tem constituído um importante obstáculo ao desenvolvimento da investigação nesta área, tanto mais que é sabido que uma boa operacionalização do conceito e métodos de avaliação bem fundamentados e com elevada validade são essenciais para reduzir a probabilidade de resultados contraditórios e inconsistentes (Allen et al., 2005; Blow \& Hartnett, 2005). Assim, foi objetivo deste estudo o desenvolvimento de um questionário de avaliação dos comportamentos de infidelidade marital, suas motivações e contextos, que enriquecesse o leque de instrumentos disponíveis neste domínio.

O desenvolvimento de um instrumento de avaliação das motivações e determinantes dos comportamentos extramaritais teria necessariamente que se basear em teorias capazes de ajudar a entender tais comportamentos. Dentro destas, por exemplo, a psicologia evolutiva tem vindo a procurar explicar as causas da infidelidade com as diferentes necessidades adaptativas de homens e mulheres, abordando as vantagens reprodutivas da infidelidade em termos de maximização da replicação dos genes (Fisher \& Cox, 2011). Assim, existirá uma predisposição inata nos homens para procurar variedade sexual, uma vez que o investimento requerido da sua parte para a reprodução é mínimo. Tendo o potencial para gerar um número praticamente ilimitado de descendentes, o sucesso reprodutivo dos homens está limitado sobretudo pelo número de parceiras sexuais disponíveis. Por isso, os homens deverão ser particularmente tentados por mulheres que se mostrem sexualmente disponíveis. Pelo contrário, para as mulheres, a variedade e a quantidade em si não seriam úteis, e o mais importante seria a obtenção dos recursos necessários para a sobrevivência dos seus descendentes (De Stefano \& Oala, 2008; Poulsen, Holman, Busby, \& Carroll, 2013). Deste modo, o principal fator limitativo do sucesso reprodutivo 
das mulheres será a disponibilidade desses recursos. Tal justificará que os homens se envolvam mais em comportamentos de infidelidade sexual e com um maior número de parceiras, tendo uma maior probabilidade de expressar esse desejo; as mulheres terão maior tendência para o envolvimento emocional com parceiros que pareçam dispostos a assegurar um elevado grau de investimento nelas e na sua descendência (Barta \& Kiene, 2005; Buss, 2008b; De Stefano \& Oala, 2008; Glass \& Wright, 1985; Munsch, 2012; Poulsen et al., 2013; Sabini \& Green, 2004).

Estas diferenças, consubstanciadas na chamada "teoria das estratégias sexuais" (Buss \& Schmitt, 1993), têm importantes implicações para a compreensão dos comportamentos de infidelidade. No caso dos homens, que tendem a usar a "estratégia a curto prazo" descrita no parágrafo anterior, parece-nos que a infidelidade poderá estar associada sobretudo a situações de tentação envolvendo mulheres atraentes e que se mostrem sexualmente disponíveis, e a motivação subjacente deverá ser sobretudo sexual. No caso das mulheres, a infidelidade deverá estar mais associada a aspetos ligados à relação primária (insatisfação, possivelmente associada à falta de vontade ou capacidade do parceiro masculino para investir recursos) e a motivação afetiva, com o objetivo de criação de uma ligação emocional com o novo parceiro, deverá ser mais importante.

A teoria da vinculação é outra abordagem potencialmente útil na explicação das causas da infidelidade, sobretudo no aspeto das diferenças individuais nos estilos de vinculação. $\mathrm{O}$ sistema de vinculação, através de comportamentos de procura de proximidade, permite um sentimento de segurança perante algum tipo de ameaça potenciadora de stress. Muitos indivíduos, porém, devido ao facto de não terem tido, ao longo do seu desenvolvimento, relações em que as suas necessidades fossem atendidas de forma sensível, não conseguem utilizar as relações próximas para alcançar essa sensação de segurança, desenvolvendo o que se designa por um estilo de vinculação inseguro. Este pode ser do tipo evitante, em que a procura de proximidade é desativada, ou do tipo ansioso/preocupado, em que é hiperativada (Mattingbaum, Weisberg, \&
Simpson, 2011; Mintz, 2004). Neste sentido, a investigação tem demonstrado que indivíduos com estilos de vinculação inseguros têm relações de casal menos harmoniosas e satisfatórias e, em muitas circunstâncias, maior número de parceiros sexuais (Hazan \& Shaver, 1994). Indivíduos com um estilo de vinculação ansioso tenderão a usar o sexo como forma de preencher as suas necessidades de autoestima e intimidade emocional (Birnbaum, Reis, Mikulincer, Gillath, \& Orpaz, 2006; Birnbaum, Simpson, Weisberg, Barnea, \& Assulin-Simhon, 2012). O estilo de vinculação evitante está associado a um menor investimento nas relações. O menor investimento, e consequente menor compromisso com o parceiro, poderão reduzir as barreiras ao envolvimento com terceiros. Além disso, o indivíduo evitante poderá utilizar a infidelidade como forma de reduzir o envolvimento emocional e aumentar o distanciamento em relação ao parceiro (Duba, Kindsvatter, \& Lara, 2008).

Outra perspetiva a ter em conta é a da motivação para a fidelidade, uma vez que esta deverá ter uma forte influência no envolvimento extrarrelacional, e a investigação tem vindo a evidenciar que diferentes motivações têm um impacto diferente na manutenção ou dissolução de uma relação (Pelletier, Tuson, \& Haddad, 1997). A teoria da autodeterminação (Deci \& Ryan, 2008) é atualmente a mais influente neste aspeto da discriminação entre tipos de motivação, sobretudo no contraste que postula entre o conceito de motivação controlada, que corresponde a uma motivação menos internalizada e traduzida em comportamentos iniciados por razões mais instrumentais, e o de motivação autónoma, mais internalizada e associada a comportamentos iniciados pela obtenção de prazer e satisfação. Estas diferenças na motivação subjacente estariam, por sua vez, relacionadas com diferentes emoções morais suscitadas pela ocorrência de comportamentos discrepantes com os princípios nela baseados (Moreira, Maia, Costa, Gonzalez, \& Santos, 2010). Assim, a culpa estaria mais associada à violação de intenções sustentadas numa motivação autónoma, enquanto a vergonha seria o sentimento predominante perante comportamentos sentidos como violadores de uma decisão fundamentada numa motivação 
mais controlada.

Outro modelo bastante influente quanto às motivações subjacentes à infidelidade é o proposto por Brown (2001a, 2001b). Baseado em dados clínicos, este modelo considera que a infidelidade tem objetivos pessoais e relacionais específicos, que podem ser de cinco tipos, nem todos eles óbvios e nem todos necessariamente contrários à manutenção da relação primária. Assim, o tipo de infidelidade que Brown (2001a, 2001b) designa por "Evitador de Conflito" surge em casais tidos como modelo, que se sacrificam pela relação e evitam o conflito e a expressão de insatisfação. Por isso, os conflitos tendem a permanecer latentes e a não ser resolvidos, funcionando a infidelidade com uma forma indireta de chamar a atenção do parceiro para os problemas da relação. No tipo "Evitador de Intimidade", o parceiro infiel tem dificuldade em lidar com a interdependência que decorre de uma relação íntima e usa a infidelidade como forma de expressar a sua individualidade e independência. No tipo designado de "Dependência Sexual", o sexo visa preencher um vazio emocional ou lidar com a dor, sendo o "affair" uma forma de conquista e de sentir poder. Pode haver também uma procura por alguém que preencha a fantasia de amor total. No tipo do "Eu Dividido", há uma tentativa de vivenciar uma individualidade emocional que terá sido negada em prol de fazer o correto, sobretudo dentro da relação primária, estando por isso esta associada a uma sensação de vazio. Este tipo é geralmente mais comum nos homens, mas nos últimos anos há mais mulheres a envolverem-se neste tipo de affairs. Finalmente, o "Affair Terminal" ocorre geralmente após a tomada de decisão de terminar a relação primária e visa sobretudo evitar assumir frontalmente a responsabilidade pelo fim desta.

Assim, tendo por base a literatura (Barta \& Kiene, 2005; Brown, 2001a, 2001b; Gillath, Mikulincer, Birnbaum, \& Shaver, 2008; Nelson, Li, Eckstein, Ane, \& Mullener, 2008; Viegas \& Moreira, 2010) e a constatação das insuficiências no modo como a infidelidade tem vindo a ser avaliada, assim como da importância de fatores pessoais e motivacionais na ocorrência de infidelidade, foi desenvolvido o Questionário Multidimensional de Comportamentos
Extrarrelacionais (QMCER), com o objetivo de caraterizar, de forma mais completa do que é comum na literatura, múltiplos aspetos como comportamentos, motivações e contextos da ocorrência de infidelidade. No estudo descrito neste artigo, a versão inicial do QMCER foi aplicada a uma amostra de participantes portugueses, juntamente com um conjunto de outros instrumentos, por forma a avaliar as suas qualidades psicométricas.

\section{Método}

\section{Participantes}

Participaram neste estudo 545 indivíduos, 149 homens (35\%) e 396 mulheres (65\%). Serão apresentados apenas os dados descritivos dos participantes que admitiram ter tido algum comportamento de infidelidade, pois só esses responderam à secção $\mathrm{E}$ do questionário QMCER, que recebe um maior destaque na análise dos resultados. Além disso, mesmo os participantes que responderam a todos os itens da secção $\mathrm{E}$ podem não ter respondido a todos os itens dos outros instrumentos, pelo que o número de sujeitos poderá ser algo inferior em algumas análises. Por razões de espaço, serão, para algumas variáveis, referidas apenas as categorias que mais se destacam, pelo que as percentagens podem não somar $100 \%$.

Assim, responderam à secção $\mathrm{E}$ do $\mathrm{QMCER}$ 156 participantes, 54 homens (35\%) e 102 mulheres $(65 \%)$. A média de idades foi de 32,99 anos $(D P=11,42)$, compreendidas entre os 18 e os 70 anos. No que concerne à escolaridade, $71 \%$ possuíam habilitações ao nível do ensino superior e $26 \%$ do ensino secundário. Somente dois participantes tinham até 6 anos de escolaridade. Havia $25 \%$ de sujeitos casados, $2 \%$ estavam noivos, $16 \%$ viviam em união de facto, $32 \%$ estavam numa relação de namoro, $22 \%$ não estavam envolvidos em nenhuma relação e $3 \%$ estavam divorciados ou separados. A duração média da relação foi de 8,71 anos $(D P=7,47)$. Uma percentagem elevada (82\%) declarou não ter tido casamentos ou uniões anteriores, $18 \%$ terá tido uma ou mais uniões anteriores. No que diz respeito à classe socioeconómica atual, 57\% dos participantes consideraram-se inseridos na classe 
média, $15 \%$ na classe média alta e $24 \%$ na classe média baixa, sendo as respostas semelhantes quando perguntado acerca da classe em que cresceram. Os católicos eram 50\%, enquanto $40 \%$ afirmaram não ter nenhuma religião. Quanto ao grau de religiosidade, avaliado numa escala de sete pontos, $82 \%$ situavam-se entre o nada religioso e o ponto médio da escala. Quanto à orientação política, $60 \%$ dos participantes considerava-se de direita, $17 \%$ ligeiramente à direita, $17 \%$ de centro e $6 \%$ de esquerda.

\section{Instrumentos}

Infidelidade. Foi utilizado o "Questionário Multidimensional de Comportamentos ExtraRelacionais" (QMCER), desenvolvido no âmbito deste estudo. Este instrumento é composto por diversas secções, cada uma delas com objetivos e conteúdos específicos:

Secção A - É perguntado ao indivíduo se está, no momento da resposta, envolvido numa relação amorosa com compromisso de exclusividade. $\mathrm{O}$ objetivo desta secção e da seguinte é apenas o de, quando utilizadas num contexto de investigação, direcionar os respondentes para as secções relevantes e excluí-los de outras. Caso o respondente responda afirmativamente nesta secção, não é apresentada a secção B.

Secção B - É perguntado ao indivíduo se já esteve, anteriormente, envolvido numa relação amorosa com compromisso de exclusividade. Se a resposta for "Sim" o participante seguirá para a secção C; caso responda "Não" é encaminhado para a secção D.

Secção C - São apresentados 10 comportamentos (e.g., "Beijei na boca de modo prolongado outra pessoa"), com o intuito de avaliar se o sujeito realizou alguns deles com pessoas que não o seu parceiro, numa altura em que estava envolvido numa relação amorosa com compromisso de exclusividade, quer seja a atual, ou uma relação passada. Procurou-se, na elaboração desta secção, incluir uma variedade de comportamentos concretos e específicos, passíveis de ocorrer numa situação de infidelidade e que representassem diferentes tipos e graus de envolvimento físico, emocional e sexual. As alternativas de resposta apresentadas eram: (a) "Não aconteceu"/ (b) "Aconteceu". "Caso este comportamento tenha ocorrido assinale todas as que se apliquem": I "Aconteceu no último ano" / II - "Aconteceu entre 1 e 5 anos" / III - "Aconteceu há mais de 5 anos" / IV - "Considero que foi uma infidelidade" / V - "Considero que não foi uma infidelidade."

Secção D - Esta secção é respondida apenas pelos indivíduos que responderam nunca ter estado envolvidos numa relação amorosa com compromisso de exclusividade, apresentando de modo hipotético os mesmos itens da secção C.

Secção E - Pretende avaliar diversos aspetos da prática de comportamentos extrarrelacionais (e.g., motivações, conhecimento por parte do parceiro), através de um conjunto de 54 itens (ver exemplos na apresentação dos resultados). Procurou-se incluir itens derivados dos possíveis mecanismos postulados pelas teorias mencionadas na introdução, bem como outros aspetos relevantes para a caracterização da situação. Esta secção apenas é respondida pelos indivíduos que indiquem, na secção $\mathrm{C}$, que tiveram um ou mais comportamentos extrarrelacionais e que os consideram como infidelidade.

Secção F - Pretende avaliar as diferentes motivações para o não envolvimento em comportamentos extrarrelacionais, ou seja, as motivações para a fidelidade (e.g., "Tenho medo das consequências para a nossa relação”). É preenchida por todos os participantes. Cada um dos 13 itens é avaliado numa escala vai de 1 , "nada verdadeira" a 7, "completamente verdadeira".

Por ser o foco deste estudo, o QMCER terá a sua análise psicométrica apresentada na secção de Resultados.

Atitudes em relação à Infidelidade. Foi utilizada a Escala de Atitudes em Relação à Infidelidade (EARI) construída por Afonso (2011), a qual pretende avaliar as atitudes negativas vs positivas face à infidelidade. Efetuou-se uma reanálise dos dados do estudo de Afonso (2011), tendo sido selecionados os quatro itens (dos 29 iniciais) com maior saturação, por forma a obter uma versão breve da escala (e.g., "A infidelidade é um modo de vida normal que não tem nada de reprovável"). É pedido aos participantes que indiquem o seu grau de concordância com as frases, numa escala de 1, 
"discordo fortemente" a 5, "concordo fortemente". O alfa de Cronbach obtido foi de .73.

Satisfação Conjugal. Foi utilizada uma versão reduzida da Relationship Rating Form (RRF; Davis \& Latty-Mann, 1987), versão portuguesa de Wolfgang Lind (Moreira et al., 2006), com os 33 itens (dos 57 originais) mais representativos dos fatores identificados nesta versão. Neste questionário, é pedido aos sujeitos que classifiquem cada um dos itens numa escala de 1, "nada" a 9, "completamente ou extremamente". Optou-se pela realização de uma análise em componentes principais para confirmar a estrutura fatorial da escala, utilizando as técnicas descritas na secção de resultados a propósito do QMCER. Assim, o Fator 1 corresponde à Confiança (e.g., "Esta pessoa pode contar consigo para o ajudar quando precisar?"), o Fator 2 à Paixão (e.g., "Dá-lhe prazer, só de observar ou olhar para esta pessoa?") e o Fator 3 ao Conflito (e.g., "Você briga e discute com esta pessoa?") No presente estudo, o alfa obtido foi de .94 na escala de Confiança, de .93 na escala de Paixão e de .89 na escala de Conflito. Cada escala era constituída por 9 itens.

Estilo de Vinculação Adulta. O questionário "Experiências em Relações Próximas" (ERP) é a versão portuguesa do "Experiences in Close Relatioships" (Brennan, Clark, \& Shaver, 1998), traduzido e adaptado por Moreira et al. (2006). Procura avaliar as duas dimensões básicas das diferenças individuais no estilo de vinculação dos adultos: a Evitação e a Preocupação. A forma reduzida utilizada neste estudo é composta por 12 itens (dos 36 iniciais), dos quais 6 medem a Evitação (e.g., "Não me sinto confortável ao 'abrir-me' com o meu parceiro/a") e 6 a Preocupação (e.g., "Fico ressentido/a quando o meu parceiro/a passa tempo longe de mim"). A escala de avaliação vai de 1 , "discordo totalmente a 7, "concordo fortemente". Foi efetuada uma análise em componentes principais, que confirmou a distribuição dos itens pelos dois fatores, tendo a escala de Evitação obtido um alfa de .75 e a de Preocupação um alfa de .78 .

Estratégias Sexuais. O Questionário de
Estratégias Sexuais-II (QES-II), desenvolvido para este estudo, é constituído por três escalas que medem a utilização de estratégias reprodutivas a curto e a longo prazo, estando as segundas subdivididas entre o investimento no parceiro/a e o investimento nos filhos. Resultou das análises efetuadas sobre a versão anterior (QES), construída por Moreira (2009). Relativamente à versão anterior, foi acrescentada a subdivisão dentro das estratégias a longo prazo, o que implicou o aumento do número de itens de 12 para 13. Os sujeitos deverão indicar o grau em que cada um dos itens corresponde aos seus comportamentos, sentimentos e atitudes, através de uma escala de Likert de 1, "Não tem a ver comigo a 7, " tem totalmente a ver comigo".

Uma análise em componentes principais permitiu a identificação de três fatores. Assim, o Fator 1 (cinco itens) corresponde à estratégia a curto prazo (e.g., "Sou infiel por natureza"), o Fator 2 (cinco itens) à estratégia a longo prazo com investimento no parceiro (e.g., "Quero que o meu marido/esposa ou companheiro/a seja o aspeto central da minha vida") e o Fator 3 (três itens) à estratégia a longo prazo com investimento nos filhos (e.g., "Tenho a intenção de investir muito tempo e esforço na relação com os meus filhos"). Refira-se que os itens 7 e 11, referentes à família em geral, saturaram mais fortemente no fator correspondente à estratégia a longo prazo com investimento no parceiro. O alfa obtido foi de .84 para a escala Estratégia a Curto Prazo; de .77 para a Estratégia a Longo Prazo com Investimento no Parceiro e de .78 para a Estratégia a Longo Prazo com Investimento nos Filhos.

Orientação Sociosexual. O Inventário de Orientação Sociosexual (IOSS; Simpson \& Gangestad, 1991) foi construído tendo como objetivo fornecer uma medida global da predisposição dos indivíduos para se envolverem em relações sexuais casuais, sem compromisso. A versão portuguesa utilizada neste estudo foi elaborada pela equipa que recolheu em Portugal os dados para o International Sexuality Description Study (Moreira, Mata, Veríssimo, Mata, \& Moreira, 2003). É composto por sete itens, dos quais quatro se referem a comportamentos sexuais envolvendo diferentes 
parceiros: dois referem-se a comportamentos passados, um a expectativas de comportamentos futuros, e outro a fantasias sexuais. Os restantes três itens referem-se a atitudes relativas ao sexo casual. A análise em componentes principais (seguindo o procedimento já descrito) permitiu verificar que os itens relativos a comportamentos e os relativos a atitudes se separavam claramente em dois fatores distintos.

Em virtude dos diferentes formatos de resposta, foi necessário proceder à padronização dos itens para se efetuar o cálculo do alfa de Cronbach. Neste cálculo, verificou-se que os itens referentes a expetativas de comportamentos futuros e a fantasias eram indicadores fracos do fator de Comportamentos, fazendo a sua eliminação aumentar o valor do coeficiente alfa, que foi de .88 para a escala de Comportamentos e de . 80 para a Escala de Atitudes.

\section{Procedimento}

Os dados foram recolhidos na Internet, tendo os questionários sido divulgados em redes sociais, sites e blogues na área da psicologia, segundo um procedimento de amostragem em "bola de neve" (Marôco, 2007). A participação dos sujeitos foi voluntária, tendo os mesmos sido informados do teor dos questionários a preencher, duração prevista da participação, confidencialidade dos dados e obrigatoriedade de ter 18 anos ou mais para poder participar. Era igualmente explicitado que, ao clicarem para prosseguir, davam o seu consentimento e garantiam que eram maiores de 18 anos.

A questão da confidencialidade assumiu particular importância, dada a temática em questão e os potenciais riscos para os participantes, pelo que foi garantido que as respostas aos questionários seriam confidenciais e que seria preservado o seu anonimato, não existindo forma de relacionar um participante com as respostas fornecidas. A investigação teve a aprovação da Comissão de Deontologia da Faculdade de Psicologia da Universidade de Lisboa.

\section{Resultados}

\section{Secções C e D: Comportamentos de infidelidade}

Optou-se por agregar, na análise, as secções C e $\mathrm{D}$, dado que o número de sujeitos que responderam à secção $\mathrm{D}$ era muito reduzido e a análise apenas da secção $\mathrm{C}$ fornecia resultados praticamente idênticos. Na presente análise apenas se considerou, para cada item (10 no total), a resposta do participante acerca de se o comportamento em causa tinha ocorrido ou não, num momento em que o participante estava envolvido numa relação de casal com compromisso de exclusividade. Foi efetuada uma análise em componentes principais dos itens destas secções, utilizando o scree plot (Moreira, 2004) como critério para a determinação do número de fatores e uma rotação do tipo Varimax.

Os três fatores encontrados pareciam estar associados ao envolvimento físico (Fator 1; e.g., "Manter relações sexuais com outra pessoa"), ao envolvimento afetivo (Fator 2: "Dizer 'amo-te' a outra pessoa") e ao desejo sexual (Fator 3: "Sentir-se sexualmente atraído por outra pessoa"). Foram derivadas destes fatores escalas compostas pelos itens com saturações superiores a .50 na matriz após rotação. A escala de Envolvimento Físico, composta por 4 itens, obteve um alfa de Cronbach de . 88, a de Envolvimento Afetivo, também com 4 itens, um alfa de .78 e a de Desejo Sexual, com 2 itens, um alfa de .81 .

\section{Secção F: Motivações para a fidelidade}

Relativamente à Secção F (composta por 13 itens), a análise em componentes principais, realizada de modo semelhante ao descrito para as secções $\mathrm{C} / \mathrm{D}$, conduziu à identificação de quatro fatores. $\mathrm{O}$ Fator 1 parece corresponder à motivação intrínseca para o não envolvimento extrarrelacional (e.g., "Amar o seu parceiro"), o Fator 2 à motivação introjectada ("Condenar a traição"), o Fator 3 à motivação extrínseca ("Ter medo de ser descoberto"), e o Fator 4 à ausência de motivação ou desejo para o envolvimento extrarrelacional ("Não conhecer ninguém que lhe desperte interesse"). A escala de Motivação Intrínseca (3 itens) obteve um alfa de Cronbach de .83 , a de Motivação Introjectada (4 itens) de .97, a Motivação Extrínseca (3 itens) de .70 e a de 
Ausência de Motivação ou Desejo (2 itens) de .50. Apesar do valor insuficiente do coeficiente alfa de Cronbach para o último fator, optámos por mantêlo, dado que corresponde a uma dimensão frequentemente encontrada em análises fatoriais de questionários de motivação e poderá ser alvo de futuros desenvolvimentos, com a adição de novos itens que aumentem a sua precisão.

\section{Secção E: Características da infidelidade}

Esta secção foi sujeita a uma análise semelhante à das secções $\mathrm{C} / \mathrm{D}$, apresentada aqui com mais detalhe, dada a sua maior complexidade. O scree plot indicou neste caso sete fatores. Razões de espaço não nos permitem apresentar a matriz completa dos fatores, que poderá ser obtida contactando os autores.

Os fatores mostraram-se claramente interpretáveis (ver designações no Quadro 1). O primeiro parece corresponder sobretudo ao aumento da autoestima como consequência do envolvimento extrarrelacional, como se pode ver pelo conteúdo dos itens que apresentam as saturações mais elevadas ("Quando penso no meu envolvimento extrarrelacional sinto-me atraente e sedutor"; "A minha autoestima aumentou depois desse envolvimento"). O segundo, pelo contrário, foca-se numa causa da infidelidade, nomeadamente a falta de satisfação na relação primária (e.g., "Envolvi-me com outra pessoa porque estava desiludido com o meu parceiro"; "Envolvi-me com outra pessoa porque estava com raiva do meu parceiro").

O terceiro fator volta a focar a relação primária, mas agora em termos das consequências da infidelidade. Neste fator predominam, então, os itens que referem um envolvimento afetivo com o parceiro secundário ("Envolvi-me com outra pessoa porque me apaixonei”), que tende a substituir neste aspeto o parceiro primário ("Envolvi-me com outra pessoa porque deixei de amar o meu parceiro"), levando à deterioração da relação com este ("A minha relação piorou depois da situação de infidelidade") e ao seu eventual término ("O meu envolvimento com outra pessoa levou ao final da minha relação"), sendo que a intenção de trocar o parceiro primário pelo secundário parece ter estado presente desde cedo nesse novo envolvimento ("Mantive uma relação com outra pessoa com a intenção de vir a ficar com ela exclusivamente").

$\mathrm{O}$ quarto fator tem claramente a ver com o conhecimento da situação por parte do parceiro primário, agregando diversas formas de obtenção desse conhecimento ("O meu parceiro soube desse envolvimento"; "O meu parceiro suspeitou desse envolvimento"). No quinto fator surgem, com as saturações mais elevadas, dois itens que correspondem a fatores situacionais na origem da infidelidade ("Envolvi-me com outra pessoa mesmo não querendo que isso acontecesse, mas foi mais forte do que eu"; "Envolvi-me com outra pessoa porque a tentação foi demasiado forte"), em que $o$ participante minimiza $o$ seu envolvimento ativo na escolha desse caminho. Os itens subsequentes, na ordenação pelas saturações, ajudam a perceber os contornos deste fator. A responsabilidade da situação é atribuída sobretudo à outra pessoa ("O envolvimento aconteceu mais por iniciativa de outra pessoa do que minha") e às suas características ("Envolvi-me com outra pessoa porque ela era muito atraente"). O envolvimento na relação secundária é desvalorizado e circunscrito ("O envolvimento extrarrelacional que mantive foi só uma fase passageira"), com algum peso no aspeto exclusivamente sexual ("Este envolvimento foi sobretudo desejo sexual"). Este envolvimento parece ser visto como contrário às inclinações do próprio, levando a sentimentos de culpa ("Quando penso nesse envolvimento sinto-me culpado"), para além de ser visto como discrepante em relação à imagem idealizada que a relação primária parece ter na rede social ("Os nossos amigos consideram a nossa relação como um modelo"; "Eu e o meu parceiro cumprimos tudo o que a família e os amigos esperavam de nós").

$\mathrm{O}$ sexto fator foca-se, mais uma vez, na relação primária, mas agora em aspetos negativos, nomeadamente o conflito ("Eu e o meu parceiro discutimos muito e de modo aceso"; "Eu e o meu parceiro não discutimos, damo-nos bem", este com saturação negativa) e em características negativas atribuídas ao parceiro ("O meu parceiro é controlador e exigente"). Finalmente, o Fator 7 parece corresponder a um padrão de dependência sexual, não só pelo elevado número de situações de envolvimento extrarrelacional ("Já tive mais de 
Quadro 1. Alfas de Cronbach e Diferenças entre Sexos nos Fatores da Secção E do QMCER

\begin{tabular}{|c|c|c|c|c|c|c|c|}
\hline \multirow[b]{2}{*}{ Fatores } & \multicolumn{2}{|c|}{ Masculino } & \multicolumn{2}{|c|}{ Feminino } & \multirow[b]{2}{*}{$F(1,154)$} & \multirow[b]{2}{*}{$p$} & \multirow[b]{2}{*}{$\alpha$} \\
\hline & $M$ & $D P$ & $M$ & $D P$ & & & \\
\hline 1. Melhoria da Autoestima & 7,78 & 5,60 & 7,17 & 5,71 & 0,41 & 0,52 & 0,92 \\
\hline 2. Insatisf. c/ a Rel. Primária & 5,50 & 4,90 & 8,21 & 6,24 & 7,65 & 0,01 & 0,86 \\
\hline 3. Affair Terminal & 3,76 & 4,09 & 5,45 & 5,05 & 4,49 & 0,04 & 0,82 \\
\hline 4. Conhecimento pelo Parceiro & 4,07 & 4,29 & 4,21 & 4,08 & 0,04 & 0,85 & 0,71 \\
\hline 5. Tentação & 3,72 & 2,22 & 3,46 & 2,32 & 0,46 & 0,50 & 0,70 \\
\hline 6. Conflito na Rel. Primária & 4,69 & 1,72 & 4,50 & 1,66 & 0,42 & 0,52 & 0,67 \\
\hline 7. Dependência Sexual & 1,98 & 2,53 & 0,76 & 1,32 & 15,62 & 0,00 & 0,59 \\
\hline
\end{tabular}

25 envolvimentos extrarrelacionais"), como pela admissão de utilizar o sexo como estratégia de regulação emocional ("Quando me sinto sozinho e/ou em sofrimento procuro sexo para fugir à dor"). Esta interpretação é apoiada também pela saturação relativamente elevada do item "Este envolvimento foi sobretudo desejo sexual" e pela saturação negativa do item "O jogo de sedução é tão ou mais importante que o envolvimento físico em si". Parece também existir, entretanto, um componente de desejo de afastamento em relação ao parceiro ("Envolvi-me com outra pessoa para poder passar menos tempo em casa").

Destes fatores foram derivadas, segundo o procedimento descrito para as secções $\mathrm{C}$ e $\mathrm{D}$, escalas que, na maioria dos casos, apresentavam valores adequados do alfa de Cronbach (Quadro 1). Para os quatro últimos fatores, porém, os alfas nestes casos são marginais ou fracos. Optou-se, ainda assim, por manter estes fatores, incluindo o Fator 7 (que apresenta um valor do alfa demasiado baixo), pela sua importância teórica e por se considerar que podem vir a ser desenvolvidos em estudos futuros.

A análise das diferenças entre sexos nas escalas desta secção revestia-se de especial interesse. O resultado global da comparação multivariada (MANOVA) foi significativo, $F(7$, $148)=4.66, p<.005$. O Quadro 1 apresenta os resultados por fator. Verificam-se diferenças significativas nos fatores de Insatisfação com a Relação Primária, Affair Terminal (em ambos os casos com médias mais altas entre as mulheres) e Dependência Sexual (com uma média mais alta entre os homens). Com o objetivo de medir a associação linear entre os fatores identificados para a secção E do QMCER e as variáveis relativas aos outros instrumentos utilizados, efetuaram-se correlações de Pearson. Estas são apresentadas separadamente para homens e mulheres, visto que são bastante diferentes em alguns casos, como se pode observar nos Quadros 2 e 3.

O Fator 1, Melhoria da Autoestima, surgiu mais fortemente associado ao envolvimento sexual e à estratégia a curto prazo e não tanto ao envolvimento afetivo. Tal verificou-se nas mulheres e nos homens, apesar de o efeito ser mais forte nos homens. Não se verificou a associação esperada com o estilo de vinculação preocupado, para nenhum dos sexos. O Fator 2, Insatisfação com a Relação Primária, nas mulheres parece estar associado à motivação extrínseca para a fidelidade e aos três níveis de envolvimento (sexual, emocional e desejo), enquanto nos homens surgiram associações com a existência de conflito e a falta de paixão e confiança na relação primária, assim como com o estilo evitante e um menor nível de investimento a longo prazo no parceiro.

O Fator 3, Infidelidade Terminal, obteve nos homens resultados muito semelhantes aos do Fator 2, sendo o conflito a variável com associação mais elevada. Nas mulheres foi possível constatar associações com a falta de confiança na relação e o envolvimento afetivo, assim como um menor investimento a longo prazo no parceiro. O Fator 4, Conhecimento do Parceiro, não apresentou nenhuma associação relevante, no que concerne às mulheres, sendo que para os homens surgiu associado a uma atitude negativa face à infidelidade e também de modo negativo à estratégia a curto prazo. Verificou-se também uma associação com a motivação controlada para a fidelidade. 
Quadro 2. Correlações entre as Características da Infidelidade (QMCER, secção C/D) e outras Variáveis, para as Mulheres

\section{Fatores}

\begin{tabular}{|c|c|c|c|c|c|c|c|c|}
\hline Instrumentos & Variáveis & $\begin{array}{l}\text { Auto- } \\
\text { Estima }\end{array}$ & $\begin{array}{c}\text { Insat. } \\
\text { Relação }\end{array}$ & $\begin{array}{l}\text { Infidel. } \\
\text { Terminal }\end{array}$ & $\begin{array}{l}\text { Conhec. } \\
\text { Parceiro }\end{array}$ & Tentação & Conflito & $\begin{array}{c}\text { Depend. } \\
\text { Sexual }\end{array}$ \\
\hline EARI & $\begin{array}{l}\text { Atitudes } \\
\text { Infidelidade }\end{array}$ & $0,30^{* *}$ & $-0,05$ & $-0,19$ & $-0,05$ & 0,07 & $-0,06$ & 0,01 \\
\hline ERP & Evitação & 0,07 & 0,11 & $0,23^{*}$ & $-0,15$ & 0,01 & 0,03 & 0,17 \\
\hline ERP & Preocupação & 0,07 & 0,13 & $-0,07$ & 0,03 & 0,09 & 0,08 & 0,17 \\
\hline QES-II & $\begin{array}{l}\text { Estratégia a Curto } \\
\text { Prazo }\end{array}$ & $0,56^{* *}$ & 0,13 & $-0,17$ & 0,05 & $0,30^{* *}$ & 0,03 & $0,43^{* *}$ \\
\hline QES-II & $\begin{array}{c}\text { Invest. Parceiro } \\
\text { Longo Prazo }\end{array}$ & $-0,15$ & $-0,03$ & $-0,30^{* *}$ & 0,00 & $-0,06$ & $-0,02$ & $-0,21^{*}$ \\
\hline QES-II & $\begin{array}{l}\text { Invest. Parental } \\
\text { Longo Prazo }\end{array}$ & $-0,04$ & $-0,09$ & $-0,10$ & $-0,06$ & $-0,14$ & 0,01 & $-0,30^{* *}$ \\
\hline $\begin{array}{l}\text { QMCER } \\
\text { Secção F }\end{array}$ & $\begin{array}{l}\text { Motiv. Autónoma } \\
\text { Fidelidade }\end{array}$ & $-0,26^{* *}$ & $-0,12$ & $-0,09$ & 0,06 & $-0,20^{*}$ & $-0,11$ & $-0,16$ \\
\hline $\begin{array}{l}\text { QMCER } \\
\text { Seçãão F }\end{array}$ & $\begin{array}{l}\text { Motiv. Controlada } \\
\text { Fidelidade }\end{array}$ & $-0,31^{* *}$ & $-0,07$ & 0,07 & 0,18 & $-0,04$ & $0,22^{*}$ & 0,02 \\
\hline $\begin{array}{l}\text { QMCER } \\
\text { Secção F }\end{array}$ & $\begin{array}{l}\text { Motiv. Extrínseca } \\
\text { Fidelidade }\end{array}$ & 0,18 & $0,31^{* *}$ & 0,09 & 0,14 & $0,22^{*}$ & $-0,01$ & $-0,01$ \\
\hline $\begin{array}{l}\text { QMCER } \\
\text { Secção F }\end{array}$ & $\begin{array}{l}\text { Amotivação } \\
\text { Fidelidade }\end{array}$ & 0,07 & 0,04 & 0,04 & 0,15 & $-0,03$ & $-0,00$ & $-0,11$ \\
\hline RRF & Confiança & $-0,14$ & $-0,20^{*}$ & $-0,33^{* *}$ & $-0,03$ & $-0,15$ & $-0,01$ & $-0,15$ \\
\hline RRF & Paixão & $-0,22^{*}$ & $-0,20$ & $-0,01$ & 0,05 & $-0,07$ & $-0,02$ & $-0,01$ \\
\hline RRF & Conflito & $0,21^{*}$ & $0,23^{*}$ & 0,03 & $-0,12$ & 0,15 & $0,25^{*}$ & $0,27^{*}$ \\
\hline IOSS & $\begin{array}{l}\text { Comportamento } \\
\text { Sexual }\end{array}$ & 0,00 & 0,01 & 0,17 & 0,09 & 0,14 & 0,04 & $0,63^{* *}$ \\
\hline IOSS & $\begin{array}{l}\text { Atitudes face ao } \\
\text { Sexo Casual }\end{array}$ & $0,30^{* *}$ & 0,04 & 0,01 & 0,10 & $0,20^{*}$ & 0,10 & $0,35^{* *}$ \\
\hline $\begin{array}{l}\text { QMCER } \\
\text { Secção C/D }\end{array}$ & $\begin{array}{l}\text { Envolvimento } \\
\quad \text { Sexual }\end{array}$ & $0,40^{* *}$ & $0,42^{* *}$ & 0,15 & 0,01 & $0,37^{* *}$ & 0,01 & 0,12 \\
\hline $\begin{array}{l}\text { QMCER } \\
\text { Secção C/D }\end{array}$ & $\begin{array}{l}\text { Envolvimento } \\
\text { Afetivo }\end{array}$ & $0,25^{*}$ & $0,40^{* *}$ & $0,47^{* *}$ & 0,07 & $0,26^{* *}$ & 0,10 & $-0,03$ \\
\hline $\begin{array}{l}\text { QMCER } \\
\text { Seç̧ão C/D }\end{array}$ & Desejo Sexual & $0,41^{* *}$ & $0,39^{* *}$ & $0,26^{* *}$ & 0,02 & $0,43^{* *}$ & 0,11 & 0,19 \\
\hline
\end{tabular}

Nota. ${ }^{*} p<0,05 ; * * p<0,01$

O Fator 5, Tentação, apresentou associações com a estratégia a curto prazo, o envolvimento sexual e o desejo sexual, sendo esta a correlação mais forte para as mulheres. Verificaram-se também associações com a motivação extrínseca para a fidelidade. O Fator 6, Conflito, surgiu Revista Iberoamericana de Diagnóstico y Evaluación - e Avaliação Psicológica. RIDEP · No42 · Vol.2 · 125-141 2016 associado, tal como seria de esperar, ao conflito na relação. Nas mulheres, verificou-se também uma associação com a motivação controlada para a fidelidade e, nos homens, com o estilo preocupado. 
Quadro 3. Correlações entre as Características da Infidelidade (QMCER, secção C/D) e outras Variáveis, para os Homens

Fatores

\begin{tabular}{|c|c|c|c|c|c|c|c|c|}
\hline Instrumentos & Variáveis & $\begin{array}{l}\text { Auto- } \\
\text { Estima }\end{array}$ & $\begin{array}{l}\text { Insat. } \\
\text { Relação }\end{array}$ & $\begin{array}{l}\text { Infidel. } \\
\text { Terminal }\end{array}$ & $\begin{array}{l}\text { Conhec. } \\
\text { Parceiro }\end{array}$ & Tentação & Conflito & $\begin{array}{c}\text { Depend. } \\
\text { Sexual }\end{array}$ \\
\hline EARI & $\begin{array}{l}\text { Atitudes } \\
\text { Infidelidade }\end{array}$ & $0,36^{* *}$ & 0,09 & $-0,18$ & $-0,34 *$ & 0,25 & 0,23 & 0,18 \\
\hline ERP & Evitação & $0,30 *$ & $0,33 *$ & $0,29 *$ & $-0,20$ & $-0,03$ & $0,29 *$ & $0,34^{*}$ \\
\hline ERP & Preocupação & $-0,07$ & 0,20 & 0,25 & 0,24 & 0,20 & $0,38 * *$ & 0,08 \\
\hline QES-II & $\begin{array}{l}\text { Estratégia a Curto } \\
\text { Prazo }\end{array}$ & $0,74 * *$ & 0,07 & $-0,10$ & $-0,32 *$ & $0,43 * *$ & 0,23 & $0,39 * *$ \\
\hline QES-II & $\begin{array}{l}\text { Invest. Parceiro } \\
\text { Longo Prazo }\end{array}$ & $-0,31 *$ & $-0,37 * *$ & $-0,32 *$ & 0,06 & 0,01 & $-0,30 *$ & $-0,15$ \\
\hline QES-II & $\begin{array}{l}\text { Invest. Parental } \\
\text { Longo Prazo }\end{array}$ & $-0,24$ & 0,04 & 0,13 & 0,14 & $-0,04$ & 0,02 & $-0,12$ \\
\hline $\begin{array}{l}\text { QMCER } \\
\text { Secção F }\end{array}$ & $\begin{array}{l}\text { Motiv. Autónoma } \\
\text { Fidelidade }\end{array}$ & $-0,29 *$ & 0,00 & $0,29 *$ & $0,29 *$ & $-0,05$ & $-0,11$ & 0,01 \\
\hline $\begin{array}{l}\text { QMCER } \\
\text { Secção F }\end{array}$ & $\begin{array}{l}\text { Motiv. Controlada } \\
\text { Fidelidade }\end{array}$ & $-0,34 *$ & $-0,02$ & 0,20 & $0,33 *$ & $-0,17$ & $-0,19$ & $-0,02$ \\
\hline $\begin{array}{l}\text { QMCER } \\
\text { Secção F }\end{array}$ & $\begin{array}{l}\text { Motiv. Extrínseca } \\
\text { Fidelidade }\end{array}$ & 0,16 & 0,06 & 0,07 & $-0,05$ & $0,37 * *$ & 0,24 & 0,13 \\
\hline $\begin{array}{l}\text { QMCER } \\
\text { Secção F }\end{array}$ & $\begin{array}{l}\text { Amotivação } \\
\text { Fidelidade }\end{array}$ & 0,00 & 0,01 & $0,28 *$ & 0,21 & 0,11 & 0,05 & 0,01 \\
\hline RRF & Confiança & 0,01 & $-0,38^{* *}$ & $-0,12$ & $-0,06$ & 0,13 & $-0,27 *$ & $-0,11$ \\
\hline RRF & Paixão & $-0,08$ & $-0,35^{* *}$ & $-0,21$ & $-0,06$ & 0,11 & $-0,09$ & 0,02 \\
\hline RRF & Conflito & $0,27 *$ & $0,51 * *$ & $0,31 *$ & 0,04 & 0,11 & $0,37 * *$ & $0,32 *$ \\
\hline IOSS & $\begin{array}{l}\text { Comportamento } \\
\text { Sexual }\end{array}$ & $0,39 * *$ & $0,40 * *$ & 0,17 & $-0,09$ & 0,27 & 0,20 & $0,54 * *$ \\
\hline IOSS & $\begin{array}{l}\text { Atitudes face ao } \\
\text { Sexo Casual }\end{array}$ & $0,55^{* *}$ & $-0,05$ & $-0,04$ & $-0,01$ & 0,22 & 0,00 & 0,05 \\
\hline $\begin{array}{l}\text { QMCER } \\
\text { Secção C/D }\end{array}$ & $\begin{array}{l}\text { Envolvimento } \\
\text { Sexual }\end{array}$ & $0,69 * *$ & 0,20 & 0,40 & $-0,04$ & $0,49 * *$ & 0,12 & 0,22 \\
\hline $\begin{array}{l}\text { QMCER } \\
\text { Secção C/D }\end{array}$ & $\begin{array}{l}\text { Envolvimento } \\
\text { Afetivo }\end{array}$ & 0,25 & 0,05 & $0,31^{*}$ & 0,03 & $0,31 *$ & 0,05 & 0,04 \\
\hline $\begin{array}{l}\text { QMCER } \\
\text { Secção C/D }\end{array}$ & Desejo Sexual & $0,30 *$ & 0,17 & $-0,02$ & $-0,09$ & $0,27 *$ & 0,26 & 0,14 \\
\hline
\end{tabular}

Nota. $* p<0,05 ; * * p<0,01$

\section{Discussão}

Os resultados deste estudo mostram que o nosso objetivo de construir um instrumento de avaliação psicológica capaz de medir diferentes facetas dos comportamentos de envolvimento extrarrelacional foi em larga medida atingido. Assim, na secção C/D, para além de terem sido derivadas escalas com alfas de Cronbach bastante elevados para as duas principais facetas do envolvimento extrarrelacional mencionadas em estudos anteriores (Allen et al.,2005), que assim ganham um reforçado fundamento, foi possível individualizar um novo fator, ausente até agora da literatura e referente ao desejo sexual independente da sua prossecução em termos comportamentais. Na secção F, foi derivada uma estrutura para os fatores ligados à motivação positiva para a fidelidade, algo que até agora, e 
tanto quanto é do nosso conhecimento, estava também ausente da literatura. A estrutura encontrada parece aproximar-se da que é proposta, nomeadamente, pela teoria da autodeterminação (Deci \& Ryan, 2008), algo que já tinha sido encontrado no estudo de Afonso (2011) com este mesmo instrumento.

Não obstante estes importantes ganhos, assim como os resultados relevantes obtidos com outros instrumentos utilizados no estudo, importa salientar aqueles que resultaram da secção $\mathrm{E}$ do QMCER e nomeadamente da sua análise fatorial, que nos permitiu identificar e avaliar diversas facetas das situações de envolvimento extrarrelacional, nem sempre devidamente valorizadas nas abordagens psicológicas a este fenómeno. Para além do triângulo relacional assim formado, existem aspetos nem sempre evidentes mas que importa ter em conta, o que procurámos evidenciar com a expressão "sete lados do triângulo", contida no título do artigo. Assim, começamos por salientar alguns aspetos relevantes dos sete fatores encontrados. O Fator 1, Melhoria da Autoestima, corresponde a um tipo de motivação frequentemente apontado no senso comum. Porém, e contrariamente ao que tem sido evidenciado por alguns autores (Birnbaum et al., 2006; Birnbaum et al., 2012), não surgiu associado ao estilo de vinculação ansioso. Pensamos que tal pode estar relacionado com o fato de a melhoria de autoestima surgir aqui, sobretudo, como consequência e não como causa da infidelidade. Além disso, esta melhoria parece prender-se com uma questão de atratividade física/sexual e com a capacidade de sedução, e não com a obtenção de proximidade emocional, mais característica do estilo ansioso/preocupado.

O Fator 2, Insatisfação com a Relação Primária, parece prender-se com níveis baixos de interações positivas como causa para a infidelidade, podendo ser associado a uma motivação mais emocional (Wilson, Mattingly, Clark, Weidler, \& Bequette, 2011). A insatisfação com a relação tem vindo a ser descrita pela literatura como uma das principais causas de infidelidade, sobretudo entre as mulheres (Carneiro et al., 2011; Nath, 2011). Os nossos resultados confirmam esta previsão, indicando que este tipo de motivação é significativamente mais elevado entre as mulheres, mas vão para além dela, ao mostrarem que, entre as mulheres mas não entre os homens, este fator está significativamente associado a um maior envolvimento nos comportamentos extrarrelacionais. Uma possibilidade a considerar seria a de este fator estar associado ao tipo de infidelidade designada por Brown (2001a, 2001b) como "eu dividido". A afirmação daquela autora de que este tipo parece estar a tornar-se mais comum entre as mulheres é coerente com a diferença de género encontrada.

O Fator 3, por seu turno, parece poder ser claramente associado ao tipo de Infidelidade Terminal proposto por Brown (2001a, 2001b). O facto de as mulheres apresentarem uma média mais elevada é coerente com a ideia da psicologia evolutiva de que os homens terão mais vantagem em manter uma multiplicidade de relações, sobretudo com envolvimento sexual, enquanto, nas mulheres, a infidelidade poderá estar mais frequentemente ligada a um objetivo de troca de parceiro. Esta interpretação é apoiada pelas correlações que se verificaram, para as mulheres, entre este fator e o envolvimento extrarrelacional afetivo, mas não com o sexual. Pelo contrário, nos homens, este fator apresenta correlações sobretudo com aspetos negativos da relação primária. Neste sentido, parece que, para as mulheres, será sobretudo o envolvimento emocional com o novo parceiro que conduz ao rompimento da relação primária, enquanto os homens terão maior propensão para tal se não estiverem satisfeitos com essa relação. Tal confirmará a ideia de que as mulheres valorizarão mais aspetos emocionais e interpessoais, enquanto os homens darão maior ênfase à satisfação das suas necessidades individuais (Birnbaum, 2007).

O Conhecimento da Infidelidade pelo Parceiro (Fator 4) parece conter em si, no caso dos homens, um aspeto protetor da relação primária, uma vez que está associado a uma atitude negativa face à infidelidade e apresenta uma associação também negativa com a estratégia a curto prazo. Assim, o conhecimento da infidelidade por parte da parceira pode ser um indicador que a troca desta por outra não é o principal objetivo. Este resultado pode refletir não só a ideia de que mesmo os homens que condenam a traição se 
envolvem em comportamentos extrarrelacionais (Foster \& Misra, 2013), como também a de que, nos homens mais do que as mulheres, o objetivo é o da manutenção simultânea das duas relações.

A Tentação (Fator 5) parece remeter sobretudo para uma motivação sexual relacionada com o contexto, mais do que com um sentimento de afastamento relativamente ao parceiro, contrariamente ao que se verifica com a Melhoria de Autoestima (Fator 1). Por outro lado, o facto de, nas mulheres, a associação entre a tentação e o desejo sexual ser mais forte do que entre a tentação e o envolvimento sexual, parece reforçar a ideia de que as mulheres têm uma menor probabilidade do que os homens de traduzir as suas motivações sexuais em comportamentos explícitos (Hackathorn et al., 2011). Outra possibilidade a considerar é a de o desejo sexual, sendo um impulso centrípeto, onde a realização da fantasia aniquilaria o desejo (Arent, 2009), acabar por funcionar como protetor da relação primária. Em qualquer caso, é provável que a diferença entre géneros nos correlatos deste fator reflita o preconceito e os tabus que ainda persistem sobre a sexualidade feminina, existindo mais barreiras para um envolvimento físico com outra pessoa fora da relação primária.

O Conflito (Fator 6), como seria de prever, surge associado à presença de conflito na relação primária. Curiosamente, as principais associações deste fator verificam-se para os homens, particularmente com o estilo de vinculação preocupado e a falta de confiança na parceira. Tal parece ir ao encontro das conclusões de Goldenberg (2013), que refere a perceção de falta de compreensão por parte das parceiras como uma das principais queixas dos homens.

A Dependência Sexual (Fator 7) surge sobretudo associada a um comportamento sexual compulsivo e à existência de vários parceiros. Assim, poderá ser associado ao tipo de infidelidade sugerido por Brown (2001a; 2001b) com essa designação. Não sendo de surpreender a sua associação, em ambos os sexos, com a estratégia sexual a curto prazo, verificam-se, nas mulheres, correlações significativas, no sentido negativo, com as estratégias a longo prazo, sugerindo que este fator, nas mulheres, corresponde em maior grau a uma estratégia reprodutiva generalizada, possivelmente ligada à adaptação a um meio social percebido como não oferecendo estabilidade relacional. Nos homens, este efeito não atinge a significância estatística, possivelmente devido a uma propensão para utilizar em simultâneo as estratégias a curto e a longo prazo. Por outro lado, nos homens, este fator surge antes associado a um estilo de vinculação evitante, o que possivelmente estará relacionado com uma tendência, por parte dos homens, a encararem o envolvimento sexual e o envolvimento emocional como estando em oposição. Deste modo, uma menor predisposição para o envolvimento emocional, característica do estilo evitante, estaria relacionada com uma maior predisposição para o envolvimento sexual.

Um dos objetivos deste estudo era o de explorar as eventuais associações entre os fatores da secção E do QMCER e a tipologia de infidelidade de Brown (2001a, 2001b). Os resultados neste aspeto são algo ambíguos. Se para alguns tipos, que mais diretamente se associam a certos comportamentos, se conseguiu fazer de forma clara essa associação (Affair Terminal e Dependência Sexual), para outros a questão é bem mais complexa. Já mencionámos a possibilidade de associar o tipo designado por "Eu Dividido" ao Fator 2, ligado à Insatisfação com a Relação Primária. Seria possível igualmente associar o Fator 6 (Conflito na relação primária) ao tipo "Evitador de Intimidade", mas o fato de não apresentar correlações claras com o estilo de vinculação evitante, que se caracteriza justamente por este tipo de atitude, joga contra essa possibilidade. Outra hipótese a considerar seria a de que o Fator 5 (Tentação) estivesse relacionado com o tipo designado por Brown como "Evitador de Conflito". A presença de associações com a imagem social positiva da relação primária (e.g., "Os nossos amigos consideram a nossa relação como um modelo"), e as correlações positivas, ainda que não significativas, com a satisfação com a relação primária apoiam esta interpretação. Estas eventuais associações, bem como outros aspetos da utilidade do modelo de Brown (2001a, 2001b) deverão ser melhor avaliadas em estudos futuros. Outras associações entre variáveis avaliadas pelo QMCER e correlatos importantes ao nível do ajustamento pessoal e relacional deveriam 
igualmente ser avaliadas por futuros estudos, por forma a enriquecer os dados sobre a validade do instrumento e as suas aplicações clínicas.

É importante reconhecer algumas limitações desta investigação, nomeadamente a discrepância entre o número de mulheres e de homens, o recrutamento por "bola de neve" e o menor número de pessoas que respondeu à secção $\mathrm{E}$, que podem ter enviesado a amostra e impedem a generalização dos resultados. $\mathrm{O}$ valor baixo do coeficiente alfa em algumas escalas pode ter dificultado a obtenção de efeitos significativos. A elevada escolaridade da amostra também poderá ter influenciado os resultados, dado que há investigações que comprovam que esta variável tem influência nas práticas sexuais e no número de parceiros das mulheres (Alvarez \& Nogueira, 2008; Sáez Uribarri \& Guijarro Santamaría, 2000), assim como no maior número de oportunidades para o envolvimento em relações extraconjugais (Allen et al., 2005). O fato de termos utilizado exclusivamente instrumentos de autorrelato também pode ter causado enviesamentos nos resultados, podendo ter conduzido a um decréscimo dos comportamentos de infidelidade assinalados, fruto da desaprovação generalizada em relação à infidelidade. Seria útil procurar levar a cabo estudos que evitassem estas limitações, e sobretudo estudos longitudinais, que demonstrassem a capacidade preditiva do QMCER e o grau de estabilidade dos seus resultados.

\section{Conclusão}

A principal inovação deste estudo prende-se com a criação do QMCER e com os fatores nele identificados, que poderão contribuir para $o$ enriquecimento da investigação sobre a temática da infidelidade. Para além da disponibilização de um instrumento de potencial utilidade para a investigação e para a clínica, este estudo apresenta avanços na clarificação dos contributos que algumas perspetivas teóricas podem dar à compreensão deste tipo de comportamentos. Nomeadamente, os resultados parecem confirmar os pressupostos da teoria evolutiva (Buss, 2008a; Schmitt, 2005a), relativamente à importância das estratégias a curto e longo prazo como alternativas adaptativas dento do repertório das estratégias reprodutivas humanas. Confirmam também a influência que os estilos de vinculação poderão ter na explicação dos comportamentos extrarrelacionais e, ainda, a utilidade de uma abordagem motivacional baseada na teoria da autodeterminação na compreensão das bases de um comportamento monogâmico. Para além disso, as associações encontradas poderão ser utilizadas pelos terapeutas na prática clínica com casais, dado que, em situações de infidelidade, conhecer as caraterísticas do affair será fundamental para auxiliar o casal a dar um entendimento ao mesmo (Brown, 2001a, 2001b), efetuando escolhas baseadas numa melhor compreensão da situação e das motivações que lhe estão subjacentes. Esperamos que, ao conseguir ver os "sete lados do triângulo", a ação do terapeuta se torne bem mais fundamentada e eficaz.

\section{Referências}

Afonso, C. (2011). Estilo de vinculação e relações extra-diádicas: Satisfação relacional e atitudes como mediadoras. Dissertação de Mestrado Integrado em Psicologia, Faculdade de Psicologia e de Ciências da Educação, Universidade de Lisboa.

Allen, E., Atkins, D., Baucom, D., Snyder, D., Gordon, K., \& Glass, S. (2005). Intrapersonal, interpersonal and contextual factors in engaging in, and responding to extramarital involvement. Clinical Psychology Science and Pratice, 12, 101-130.

Alvarez, M. J., \& Nogueira, J. (2008). Definições sexuais de estudantes universitários. Psicologia, 23, 59-76.

Arent, M. (2009). (In)fidelidade feminina: Entre a fantasia e a realidade. Psicologia Clínica, 21, 153-167.

Barta, W. D., \& Kiene, M. (2005). Motivations for infidelity in heterosexual dating couples: The roles of gender, personality differences, and sociosexual orientation. Journal of Social and Personal Relationships 22, 339-360.

Birnbaum, G. (2007). Beyond the borders of reality: Attachment orientations and sexual fantasies. Personal Relationships, 14, 321- 
342.

Birnbaum, G., Reis, H., Mikulincer, M., Gillath, O., \& Orpaz, A. (2006). When sex is more than just sex: Attachment orientations, sexual experience, and relationship quality. Journal of Personality and Social Psychology, 91, 929-943.

Birnbaum, G., Simpson, J., Weisberg, Y.J., Barnea, E., \& Assulin-Simhon, Z. (2012). Is it my overactive imagination? The effects of contextually activated attachment insecurity on sexual fantasies. Journal of Social and Personal Relationships, 29, 1131-1152.

Birnbaum, G., Weisberg, Y. J., \& Simpson, J. A. (2011). Desire under attack: Attachment orientations and the effects of relationship threat on sexual motivations. Journal of Social and Personal Relationships 28, 448468.

Blow, A. J., \& Hartnett, K. (2005). Infidelity in committed relationships I: A methodological review. Journal of Marital and Family Therapy, 31, 183-216.

Boekhout, B., Hendrick, S., \& Hendrick, C. (2003). Exploring infidelity: Developing the relationship issues scale. Journal of Loss and Trauma, 8, 283-306.

Brennan, K. A., Clark, C. L., \& Shaver, P. R. (1998). Self-report measurement of adult romantic attachment: An integrative overview. In J. A. Simpson \& W. Rholes (Eds.), Attachment theory and close relationships (pp. 46-76). New York: Guilford.

Brown, E. M. (2001a). Affairs: Um guia para sobreviver às repercussões da infidelidade (I. Sá \& M. J. Alvarez, Trad.). Lisboa: Sílabo.

Brown, E. M. (2001b). Patterns of infidelity and their treatment $\left(2^{\text {nd }}\right.$ ed.). Philadelphia, PA, USA: Brunner-Routledge.

Buss, D. M. (2008a). Evolutionary psychology: The new science of the mind. Boston, MA, USA: Pearson.

Buss, D. (2008b). Attractive women want it all: Good genes, economic investment, parenting proclivities, and emotional commitment. Evolutionary Psychology, 6, 134-146.

Buss, D. M., \& Schmitt, D. P. (1993). Sexual strategies theory: An evolutionary perspective on human mating. Psychological Review, 100, 204-232.

Carneiro, C., Martini, C., Rodrigues, E., Oliveira, M., Romero, N., \& Oliveira, R. (2009). Infidelidade feminina na visão de homens e mulheres: Um estudo à luz da teoria das representações sociais e de género. Psicologia IESB, 1, 34-41.

Davis, K. E., \& Latty-Mann, H. (1987). Love styles and relationship quality: A contribution to validation. Journal of Social and Personal Relationships, 4, 409-428.

Deci, E., \& Ryan, R. (2008). Self-determination theory: A macrotheory of human motivation, development, and health. Canadian Psychology, 49, 182-185.

De Stefano, J., \& Oala, M. (2008). Extramarital affairs: Basic considerations and essential tasks in clinical work. The Family Journal, $16,13-19$.

Duba, J., Kindsvatter, A., \& Lara, T. (2008). Treating infidelity: Considering narratives of attachment. The Family Journal, 16, 293-299.

Fife, S., Weeks, G., \& Gambescia, N. (2008). Treating infidelity: An integrative approach. The Family Journal, 16, 316-323.

Fisher, M., \& Cox, A. (2011). Four strategies used during intrasexual competition for mates. Personal Relationships, 18, 20-38.

Foster, J. D., \& Misra, T. A. (2013). It did not mean anything (about me): Cognitive dissonance theory and the cognitive and affective consequences of romantic infidelity. Journal of Social and Personal Relationships, $30,835-857$.

Gillath, O., Mikulincer, M., Birnbaum, G. E., \& Shaver, P.R. (2008). When sex primes love: Subliminal sexual priming motivates relationship goal pursuit. Personality and Social Psychology Bulletin, 34, 1057-1069.

Glass, S. P., \& Wright, T. L. (1985). Sex differences in types of extramarital involvement and marital dissatisfaction. Sex Roles, 12, 1101-1119.

Goldenberg, M. (2013). Relações amorosas e conjugalidades: Amor, casamento e fidelidade na cultura brasileira. Gênero na Amazónia, 3, 13-24.

Hackathorn, J., Mattingly, B., Clark, E., \& 
Mattingly, M. (2011). Practicing what you preach: Infidelity attitudes as a predictor of fidelity. Current Psychology, 30, 299-311.

Hall, J. H., \& Fincham, F. D. (2006). Relationship dissolution following infidelity: The roles of attributions and forgiveness. Journal of Social and Clinical Psychology, 25, 508-522.

Hall, J. H., \& Fincham, F. D. (2009). Psychological distress: Precursor or consequence of dating infidelity? Personality and Social Psychology Bulletin, 35, 143-159.

Hazan, C., \& Shaver, P.R. (1994). Attachment as an organizational framework for research on close relationships. Psychological Inquiry, 5, $1-22$.

Liu, C. (2000). A theory of marital sexual life. Journal of Marriage and Family, 62, 363-374.

Magalhães, M. (2009). A infidelidade conjugal e seus mitos: Uma leitura gestáltica. IGT na Rede, 6, 58-90.

Maroco, J. (2007). Análise estatística com utilização do SPSS. Lisboa: Sílabo.

Mintz, A., S. (2004). Vinculação, casal e família. In N. Guedeney \& A. Guedeney (Coord.), Vinculação: Conceitos e Aplicações (pp. 183189). Lisboa: Climepsi.

Moreira, J. M. (2004). Questionários: Teoria e prática. Coimbra: Almedina.

Moreira, J. M. (2009). Estilo de vinculação adulta como mediador da relação entre experiências parentais na infância e estratégias sexuais na idade adulta. Dados não publicados.

Moreira, J. M., Mata, A., Veríssimo, J., Mata, R., \& Moreira, S. (2003, Outubro). O que se faz por cá: Resultados Portugueses do "International Sexuality Description Project". Simpósio apresentado no V Simpósio Nacional de Investigação em Psicologia, Lisboa.

Moreira, J. M., Lind, W., Santos, M. J., Moreira, A. R., Gomes, M. J., Justo, J., Oliveira, A. P., Filipe, L. A., \& Faustino, M. (2006). "Experiências em relações Próximas"; um questionário de avaliação das dimensões básicas dos estilos de vinculação nos adultos. Tradução e validação para a população portuguesa. Laboratório de Psicologia, 4, 327.

Moreira, J. M., Maia, A., Costa, C., Gonzalez, D.,
\& Santos, A. (2010). O papel das emoções de vergonha e culpa no comportamento moral: A importância das motivações subjacentes. Ousar Integrar: Revista de Reinserção Social e Prova, 7, 1-12.

Munsch, C. (2012). The science of two timing: The state of infidelity research. Sociology Compass, 6, 46-59.

Nath, S. (2011). What makes people infidel? An analysis of the influence of demographics on extramarital affairs. Undergraduate Economic Review, 8, 1-17.

Nelson, J. A., Li, C., Eckstein, D. G., Ane, P., \& Mullener, W. (2008). Antidotes for infidelity and prescriptions for long lasting relationships: Four couples activities. The Family Journal, 16, 375-378.

Pelletier, L., Tuson, K., \& Haddad, N. (1997). Client motivation for therapy scale: A measure of intrinsic motivation, extrinsic motivation and amotivation for therapy. Journal of Personality Assessment, 68, 414435.

Poulsen, F., Holman, T., Busby, D., \& Carroll, J. (2013). Physical attraction, attachment styles and dating development. Journal of Social and Personal Relationships, 30, 301-319.

Previti, D., \& Amato, P. (2004). Is infidelity a cause or a consequence of poor marital quality? Journal of Social and Personal Relationships, 21, 217-230.

Romero Palencia, A., Rivera Aragón, S., \& Díaz Loving. R. (2007). Desarrollo del Inventario Multidimensional de Infidelidad (IMIN). Revista Iberoamericana de Diagnóstico y Evaluación - e Avaliação Psicológica, 23, 121-147.

Rubin, Z. (1970). Measurement of romantic love. Journal of Personality and Social Psychology, $16,265-273$.

Sabini, J., \& Green, M. (2004). Emotional responses to sexual and emotional infidelity: Constants and differences across genders, samples and methods. Personality and Social Psychology Bulletin, 30, 1375-1388.

Sáez Uribarri, I., \& Guijarro Santamaría, C. (2000). Actitudes y experiencia sexual en mujeres jóvenes. Revista Iberoamericana de Diagnóstico y Evaluación - e Avaliação 
Psicológica, 9, 73-90.

Schmitt, D. P. (2005). Is short term mating the maladaptive result of insecure attachment? A test of competing evolutionary perspectives. Personality and Social Bulletin, 20, 1-23.

Simpson, J. A., \& Gangestad, S. W. (1991). Individual differences in sociosexuality: Evidence for convergent and discriminant validity. Journal of Personality and Social Psychology, 60, 870-883.

Snyder, D. K., Baucom, D. H., \& Gordon, K. C. (2008). An integrative approach to treating infidelity. The Family Journal, 16, 300-307.

Treas, J., \& Giesen, D., (2000). Sexual infidelity among married and cohabiting Americans. Journal of Marriage and Family, 62, 48-60.

Viegas, T., \& Moreira, J. M. (2010). Julgamentos de infidelidade: Um estudo exploratório dos seus determinantes. Estudos de Psicologia, $18,411-418$.

Wilson, K., Mattingly, B., Clark, E., Weidler, D., $\&$ Bequette, A. (2011). The gray area: Exploring attitudes toward infidelity and the development of the perceptions of dating infidelity scale. Journal of Social Psychology, 151, 63-86. 